\title{
On the classification of abelian extensions
}

\author{
Paul Bressler \\ Departamento de Matemáticas \\ Universidad de los Andes, Bogotá, Colombia \\ E-mail address: paul.bressler@gmail.com
}

\begin{abstract}
For a flat Lie algebra $\mathfrak{g}$ and a $\mathfrak{g}$-module $M$ in a symmetric monoidal abelian category linear over a field of characteristic zero we establish an equivalence of categories between the groupoid of Lie algebra extensions of $\mathfrak{g}$ by $M$ and the groupoid of extensions in the category of $\mathfrak{g}$-modules of the augmentation ideal $U(\mathfrak{g})+$ by $M$.
\end{abstract}

\section{Introduction}

In this note we give an "explicit" classification of abelian extensions of Lie algebras. Of course, the classification problem for abelian extensions has a well-known solution, at least at the level of the set of isomorphism classes, in the setting of, say, vector spaces over a field, which can be found, for example, in [CE]. Namely, it is well-known that that there is a natural bijection

$$
\pi_{0}(\operatorname{AbExt}(\mathfrak{g}, M)) \stackrel{\cong}{\longrightarrow} H^{2}(\mathfrak{g} ; M) .
$$

Here and below, $\operatorname{AbExt}(\mathfrak{g}, M)$ denotes the groupoid of abelian extensions of the Lie algebra $\mathfrak{g}$ by the $\mathfrak{g}$-module $M$ (this notion is recalled below), so that $\pi_{0}(\operatorname{AbExt}(\mathfrak{g}, M))$ is the set of isomophism classes of such.

The goal of this note is to lift (1) to an equivalence of groupoids. The traditional construction of the bijection (1) uses the realization of $H^{2}(\mathfrak{g} ; M)$ as the (degree two) cohomology of the Chevalley-Eilenberg complex $C^{\bullet}(\mathfrak{g} ; M)$ and and additional datum of a splitting of (3) to construct a cocycle in $C^{2}(\mathfrak{g} ; M)$. In our approach we interpret Lie algebra cohomology according to Yoneda, i.e. in terms of extensions in the category of $\mathfrak{g}$-modules, and utilize only "natural" constructions which do not depend on additional choices

1991 Mathematics Subject Classification. 17B56.

Key words: Lie algebra, abelian extension, tensor category.

Partially supported by FAPESP, Processo 2010/16891-3. 
and apply in our more general setting of a strict symmetric monoidal closed abelian category $\mathcal{C}$ linear over a field $k$ of characteristic zero which admits certain colimits which behave well with respect to the monoidal structure. Categories of sheaves of $k$-vector spaces furnish a large family of examples we have in mind.

Notions of a Lie algebra, of a module, of an abelian extension and of the universal enveloping algebra make sense in $\mathcal{C}$. The map

$$
\pi_{0}(\operatorname{AbExt}(\mathfrak{g}, M)) \rightarrow H^{2}(\mathfrak{g} ; M)=\operatorname{Ext}_{U(\mathfrak{g})}^{2}(\mathbb{1}, M),
$$

where $\mathbb{1}$ denotes the unit object in $\mathcal{C}$, still exists in this setting, but it is neither injective nor surjective in general as will be explained below.

As indicated above, the classification problem at hand concerns a groupoid (namely, $\operatorname{AbExt}(\mathfrak{g}, M)$ ), while the appearance of $H^{2}(\mathfrak{g} ; M)$ is suggestive of a higher order structure. Hence, one is persuaded to replace $H^{2}(\mathfrak{g} ; M)$ by a suitable Ext ${ }^{1}$-group. This idea goes back to [CE] where a bijection $\operatorname{Ext}_{U(\mathfrak{g})}^{1}\left(U(\mathfrak{g})_{+}, M\right) \rightarrow \pi_{0}(\operatorname{AbExt}(\mathfrak{g}, M))$ is established.

In fact, as it turns out, the map (2), although defined under very general circumstances, cannot be expected to be an isomorphism. Instead, the short exact sequence of $U(\mathfrak{g})$-modules

$$
0 \rightarrow U(\mathfrak{g})_{+} \rightarrow U(\mathfrak{g}) \rightarrow \mathbb{1} \rightarrow 0
$$

gives rise to the map (connecting homomorphism)

$$
\delta: \operatorname{Ext}_{U(\mathfrak{g})}^{1}\left(U(\mathfrak{g})_{+}, M\right) \rightarrow H^{2}(\mathfrak{g} ; M)
$$

which is not an isomorphism in general, but is part of a long exact sequence:

$$
\begin{aligned}
& \cdots \rightarrow \operatorname{Ext}_{U(\mathfrak{g})}^{1}(U(\mathfrak{g}), M) \rightarrow \operatorname{Ext}_{U(\mathfrak{g})}^{1}\left(U(\mathfrak{g})_{+}, M\right) \stackrel{\delta}{\rightarrow} \\
& H^{2}(\mathfrak{g} ; M) \rightarrow \operatorname{Ext}_{U(\mathfrak{g})}^{2}(U(\mathfrak{g}), M) \rightarrow \cdots
\end{aligned}
$$

Under mild assumptions on $\mathcal{C}$ one has the (canonical) isomorphisms $\operatorname{Ext}_{U(\mathfrak{g})}^{i}(U(\mathfrak{g}), M) \cong \operatorname{Ext}_{\mathcal{C}}^{i}(\mathbb{1}, M)$, hence the exact sequence

$$
\begin{aligned}
\cdots \rightarrow \operatorname{Ext}_{\mathcal{C}}^{1}(\mathbb{1}, M) \rightarrow \operatorname{Ext}_{U(\mathfrak{g})}^{1}\left(U(\mathfrak{g})_{+}, M\right) \stackrel{\delta}{\rightarrow} \\
\quad H^{2}(\mathfrak{g} ; M) \rightarrow \operatorname{Ext}_{\mathcal{C}}^{2}(\mathbb{1}, M) \rightarrow \cdots
\end{aligned}
$$

showing that the failure of the connecting homomorphism $\delta$ to be an isomorphism is due to nontrivial extensions in $\mathcal{C}$.

Let $\mathbb{E} \mathbb{X} \mathbb{T}_{U(\mathfrak{g})}^{1}\left(U(\mathfrak{g})_{+}, M\right)$ denote the groupoid of extensions of $U(\mathfrak{g})_{+}$by $M$ in the category of $U(\mathfrak{g})$-modules. Then, by definition $\operatorname{Ext}_{U(\mathfrak{g})}^{1}\left(U(\mathfrak{g})_{+}, M\right)=\pi_{0}\left(\mathbb{E} \mathbb{X}_{U(\mathfrak{g})}^{1}\left(U(\mathfrak{g})_{+}, M\right)\right)$. 
The main results of this note, the constructions of Section 3 and Theorem 3.3 , establish a natural equivalence of groupoids

$$
\operatorname{AbExt}(\mathfrak{g}, M) \cong \mathbb{E} \mathbb{X} \mathbb{T}_{U(\mathfrak{g})}^{1}\left(U(\mathfrak{g})_{+}, M\right)
$$

It should be pointed out that the train of thought involving $\operatorname{Ext}_{U(\mathfrak{g})}^{1}\left(U(\mathfrak{g})_{+}, M\right)$ is already present in [CE]. The constructions of Section 3 seems to be new.

1.1. Acknowledgements. The author would like to thank Max-PlanckInstitut für Mathematik and Instituto de Matemática e Estatistica of the University of Sao Paulo for hospitality.

\section{Preliminaries}

In what follows we work in an abelian category $\mathcal{C}$, linear over a field of characteristic zero, equipped with a strict symmetric monoidal closed structure $\otimes: \mathcal{C} \times \mathcal{C} \rightarrow \mathcal{C}$ with the unit object denoted $\mathbb{1}$. We assume that countable coproducts in $\mathcal{C}$ are representable and that $\otimes$ preserves countable colimits. An object $A$ of $\mathcal{C}$ is called flat if the functor $A \otimes \bullet: \mathcal{C} \rightarrow \mathcal{C}$ is exact.

2.1. Lie algebras. The notion of a Lie algebra in $\mathcal{C}$ makes sense: it an object $\mathfrak{g} \in \mathcal{C}$ together with a morphism

$$
[,]: \mathfrak{g} \otimes \mathfrak{g} \rightarrow \mathfrak{g}
$$

which is skew-symmetric, i.e. $[]=,(-1) \cdot[,] \circ \tau$ (where $\tau$ denote the symmetry) and satisfies the Jacobi identity

$$
[,] \circ(\operatorname{Id} \otimes[,])=[,] \circ([,] \otimes \operatorname{Id})+[,] \circ(\operatorname{Id} \otimes[,] \circ(\tau \otimes I d)
$$

A Lie algebra is called abelian if the structure morphism (the bracket) is trivial.

A morphism of Lie algebras $\varphi:\left(\mathfrak{g}_{1},[,]_{1}\right) \rightarrow\left(\mathfrak{g}_{1},[,]_{1}\right)$ is a morphism $\varphi: \mathfrak{g}_{1} \rightarrow \mathfrak{g}_{1}$ in $\mathcal{C}$ such that that the diagram

is commutative.

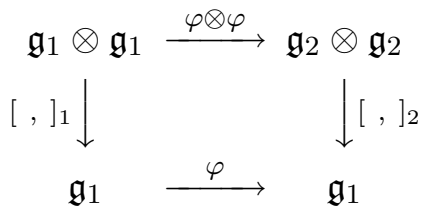

For a Lie algebra $\mathfrak{g}$ as above, a $\mathfrak{g}$-module is an object $M$ together with the morphism

$$
\alpha: \mathfrak{g} \otimes M \rightarrow M
$$


which satisfies

$$
\alpha \circ(\operatorname{Id} \otimes \alpha)=\alpha \circ([,] \otimes I d)+\alpha \circ(\tau \otimes I d)
$$

2.2. Lie algebra extensions. An extension of a Lie algebra $(\mathfrak{g},[]$,$) is a$ Lie algebra $\left(\widehat{\mathfrak{g}},[,]^{\top}\right)$ together with an epimorphism $\pi: \widehat{\mathfrak{g}} \rightarrow \mathfrak{g}$ which is a morphism of Lie algebras. Since $\operatorname{ker}(\pi)$ is an ideal in $\widehat{\mathfrak{g}}$, it is a $\widehat{\mathfrak{g}}$-module (a submodule of the adjoint representation of $\widehat{\mathfrak{g}}$. The extension $\pi: \widehat{\mathfrak{g}} \rightarrow \mathfrak{g}$ is called abelian if $\operatorname{ker}(\pi)$ is an abelian subalgebra of $\widehat{\mathfrak{g}}$. In the latter case, the $\widehat{\mathfrak{g}}$-module structure on $\operatorname{ker}(\pi)$ is induced from a unique $\mathfrak{g}$-module structure (by restriction via $\pi$ ).

Suppose given a $\mathfrak{g}$-module $M$ which will be considered as an abelian Lie algebra. An (abelian) extension of $\mathfrak{g}$ by $M$ is an extension $\pi: \widehat{\mathfrak{g}} \rightarrow \mathfrak{g}$ of $\mathfrak{g}$ together with a morphism $\iota: M \rightarrow \widehat{\mathfrak{g}}$ in $\mathcal{C}$ such that the sequence

$$
0 \rightarrow M \stackrel{\iota}{\rightarrow} \widehat{\mathfrak{g}} \stackrel{\pi}{\rightarrow} \mathfrak{g} \rightarrow 0
$$

is exact, and the $\widehat{\mathfrak{g}}$-module structure on $M$ induced via $\pi$ coincides with that of a submodule of the adjoint representation of $\widehat{\mathfrak{g}}$.

A morphism $\varphi:\left(\widehat{\mathfrak{g}}_{1}, \pi_{1}, \iota_{1}\right) \rightarrow\left(\widehat{\mathfrak{g}}_{2}, \pi_{2}, \iota_{2}\right)$ of extensions of $\mathfrak{g}$ by $M$ is a morphism of Lie algebras $\varphi: \widehat{\mathfrak{g}}_{1} \rightarrow \widehat{\mathfrak{g}}_{2}$ such that $\varphi \circ \iota_{1}=\iota_{2}$ and $\pi_{2} \circ \varphi=\pi_{1}$. Such a morphism is automatically an isomorphism.

Let $\operatorname{AbExt}(\mathfrak{g}, M)$ denote the category whose objects are extensions of $\mathfrak{g}$ by $M$, and the morphisms are as defined above. Note that $\operatorname{AbExt}(\mathfrak{g}, M)$ is a groupoid. The operation of Baer sum of extensions induces a structure of a Picard groupoid on $\operatorname{AbExt}(\mathfrak{g}, M)$.

2.3. Universal enveloping algebra. In what follows we assume that the Lie algebra $\mathfrak{g}$ and the $\mathfrak{g}$-module $M$ under consideration are flat objects in $\mathcal{C}$. We will work with the universal enveloping algebra $U(\mathfrak{g})$ and the category of $U(\mathfrak{g})$-modules. The former is an associative algebra in $\mathcal{C}$ defined as the cokernel of the map

$$
\mathrm{T}(\mathfrak{g}) \otimes \mathrm{T}^{2}(\mathfrak{g}) \otimes \mathrm{T}(\mathfrak{g}) \rightarrow \mathrm{T}(\mathfrak{g})
$$

where, for an object $A, \mathrm{~T}^{0}(A)=\mathbb{1}, \mathrm{T}^{i}(A)=A^{\otimes i}$ for $i>0, \mathrm{~T}(A)=$ $\coprod_{i=0}^{\infty} \mathrm{T}^{i}(A)$, and the map is given by $m^{(3)} \circ(\operatorname{Id} \otimes(j-j \circ \tau-[],) \otimes \operatorname{Id})$, where $m^{(3)}: \mathrm{T}(\mathfrak{g}) \otimes \mathrm{T}(\mathfrak{g}) \otimes \mathrm{T}(\mathfrak{g}) \rightarrow \mathrm{T}(\mathfrak{g})$ is the multiplication and $j: \mathrm{T}^{2}(\mathfrak{g}) \rightarrow \mathrm{T}(\mathfrak{g})$ is the inclusion. Thus defined, $U(\mathfrak{g})$ is an associative algebra object in $\mathcal{C}$ with unit (a morphism 1: $\mathbb{1} \rightarrow U(\mathfrak{g})$ equal to the composition $\mathbb{1} \rightarrow$ $\mathrm{T}^{0}(\mathfrak{g}) \rightarrow \mathrm{T}(\mathfrak{g}) \rightarrow U(\mathfrak{g})$. The projection $\mathrm{T}(\mathfrak{g}) \rightarrow \mathrm{T}^{0}(\mathfrak{g})=\mathbb{1}$ descends to the augmentation (a map of algebras) $U(\mathfrak{g}) \rightarrow \mathbb{1}$ which splits the unit so that $U(\mathfrak{g})=U(\mathfrak{g})_{+} \oplus \mathbb{1}$. 
A $U(\mathfrak{g})$-module is an object $M$ together with a morphism $\alpha: U(\mathfrak{g}) \otimes M \rightarrow$ $M$ which satisfies the usual properties. Together with morphisms defined in the obvious way $U(\mathfrak{g})$-modules form an abelian category equivalent to that of $\mathfrak{g}$-modules.

Let $\mathbb{E} \mathbb{X T}_{U(\mathfrak{g})}^{1}\left(U(\mathfrak{g})_{+}, M\right)$ denote the category of extensions of $U(\mathfrak{g})_{+}$by $M$ in $U(\mathfrak{g})$-modules. The objects of $\mathbb{E} \mathbb{X T}_{U(\mathfrak{g})}^{1}\left(U(\mathfrak{g})_{+}, M\right)$ are triples $(E, \sigma, \kappa)$ corresponding to extensions of the form

$$
0 \rightarrow M \stackrel{\kappa}{\rightarrow} E \stackrel{\sigma}{\rightarrow} U(\mathfrak{g})_{+} \rightarrow 0
$$

A morphism $\vartheta:\left(E_{1}, \sigma_{1}, \kappa_{1}\right) \rightarrow\left(E_{2}, \sigma_{2}, \kappa_{2}\right)$ of extensions is a morphism $\vartheta: E_{1} \rightarrow E_{2}$ of $U(\mathfrak{g})$-modules such that $\vartheta \circ \kappa_{1}=\kappa_{2}$ and $\sigma_{2} \circ \vartheta=\sigma_{1}$. Such a morphism is automatically an isomorphism, i.e. $\mathbb{E X}_{U(\mathfrak{g})}^{1}\left(U(\mathfrak{g})_{+}, M\right)$ is a groupoid. In fact, it is a Picard groupoid under the operation of Baer sum of extensions.

\section{Constructions}

In what follows we assume that $\mathfrak{g}$ is a flat Lie algebra and $M$ is a flat $\mathfrak{g}$-module.

3.1. From $\operatorname{AbExt}(\mathfrak{g}, M)$ to $\mathbb{E X T}_{U(\mathfrak{g})}^{1}\left(U(\mathfrak{g})_{+}, M\right)$. Suppose given an (abelian) extension $(\widehat{\mathfrak{g}}, \pi, \iota)$ of $\mathfrak{g}$ by $M$ as in (3). Let $\mathfrak{G}$ denote the cone of the morphism $\iota$. Thus, $\mathfrak{G}$ is a complex concentrated in degrees -1 and 0 , with $\mathfrak{G}^{-1}=M, \mathfrak{G}^{0}=\widehat{\mathfrak{g}}$ and the only non-trivial differential given by the map $\iota$.

The complex $\mathfrak{G}$ has a canonical structure of a differential-graded Lie algebra (DGLA) with the non-trivial components of the bracket given by

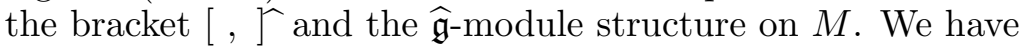

- the quasi-isomorphism of DGLA $\pi: \mathfrak{G} \rightarrow \mathfrak{g}$ induced by the morphism $\pi$ of (3),

- the morphism of DGLA $i: \widehat{\mathfrak{g}} \rightarrow \mathfrak{G}$ (inclusion of the degree-zero component),

- the morphism of graded Lie algebras $j: M[1] \rightarrow \mathfrak{G}$

The morphism $\pi$ induces the quasi-isomorphism of differential-graded algebras denoted $\pi: U(\mathfrak{G}) \rightarrow U(\mathfrak{g})$. Therefore, we have the exact sequence

$$
\cdots \rightarrow U(\mathfrak{G})^{-2} \stackrel{\partial^{-2}}{\longrightarrow} U(\mathfrak{G})^{-1} \stackrel{\partial^{-1}}{\longrightarrow} U(\mathfrak{G})^{0} \stackrel{\pi}{\rightarrow} U(\mathfrak{g}) \rightarrow 0
$$

The morphism $i$ induces the isomorphism $i: U(\widehat{\mathfrak{g}}) \rightarrow U(\mathfrak{G})^{0}$ which we will use to identify the former with the latter. 
The exact sequence (5) gives, upon passing to the augmentation ideals and using the above identification, the short exact sequence

$$
0 \rightarrow \operatorname{coker}\left(\partial^{-2}\right) \stackrel{\partial^{-1}}{\longrightarrow} U(\widehat{\mathfrak{g}})_{+} \stackrel{\pi}{\longrightarrow} U(\mathfrak{g})_{+} \rightarrow 0
$$

The morphism $j: M[1]=\mathfrak{G}^{-1} \rightarrow U(\mathfrak{G})^{-1}$ and the $U(\widehat{\mathfrak{g}})$-module structure on $U(\mathfrak{G})^{-1}$ give rise to the isomorphism $\alpha: U(\widehat{\mathfrak{g}}) \otimes M[1] \rightarrow U(\mathfrak{G})^{-1}$. On the other hand, the adjoint action of $\widehat{\mathfrak{g}}$ on $M$ gives rise to the map ad $: U(\widehat{\mathfrak{g}}) \otimes M[1] \rightarrow M[1]$. Let

$$
\delta: U(\mathfrak{G}) \rightarrow M[1]
$$

denote the map whose only non-trivial component $\delta^{-1}$ satisfies $\delta^{-1} \circ \alpha=$ ad.

Lemma 3.1. The morphism (7) is a morphism of complexes, i.e. $\delta^{-1} \circ$ $\partial^{-2}=0$.

It follows from Lemma 3.1 that the map $\delta^{-1}$ factors canonically through $\operatorname{coker}\left(\partial^{-2}\right)$; we denote the induced map coker $\left(\partial^{-2}\right) \rightarrow M$ by $\delta^{-1}$ as well.

We define $(E, \kappa, \sigma)=\mathrm{E}(\mathfrak{g}, \pi, \iota)$ as the push-out of $(6)$ by $\delta^{-1}$ :

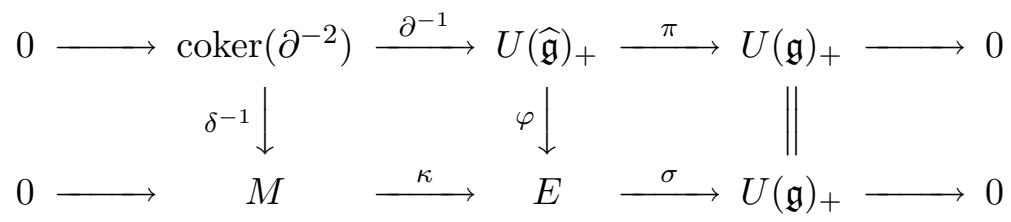

We claim that the subalgebra $M$ of $\widehat{\mathfrak{g}}$ annihilates $E$. We will show this pretending that our objects have underlying sets and leave the diagrammatic formalities to the interested reader. Suppose that $m \in M$ and $x \in \widehat{\mathfrak{g}}$. We have $m x=x m-[x, m]=\partial^{-1}(x \otimes m-1 \otimes[x, m])$. Since $\delta^{-1}(x \otimes m-1 \otimes[x, m])=0$, it follows that the image of $m x$ in $E$ is equal to zero as well.

It is clear that the assignment $(\mathfrak{g}, \pi, \iota) \mapsto \mathrm{E}(\mathfrak{g}, \pi, \iota)$ extends to a functor

$$
\mathrm{E}: \operatorname{AbExt}(\mathfrak{g}, M) \rightarrow \mathbb{E} \mathbb{X}_{U(\mathfrak{g})}^{1}\left(U(\mathfrak{g})_{+}, M\right)
$$

3.2. From $\mathbb{E} \mathbb{T}_{U(\mathfrak{g})}^{1}\left(U(\mathfrak{g})_{+}, M\right)$ to $\operatorname{AbExt}(\mathfrak{g}, M)$. Suppose given an extension of $U(\mathfrak{g})_{+}$by $M$ in the category of $U(\mathfrak{g})$-modules of the form (4).

Let $\mu: U(\mathfrak{g}) \otimes U(\mathfrak{g}) \rightarrow U(\mathfrak{g})$ and $\alpha: U(\mathfrak{g}) \otimes E \rightarrow E$ denote the algebra and the module structure morphisms respectively. We define the map $\mu_{E}$ : $E \otimes E \rightarrow E$ as the composition

$$
E \otimes E \stackrel{\sigma \otimes \mathrm{Id}}{\longrightarrow} U(\mathfrak{g})_{+} \otimes E \stackrel{\alpha}{\longrightarrow} E
$$

Lemma 3.2. The map (10) enjoys the following properties: 
(1) $\sigma \circ \mu_{E}=\mu \circ(\sigma \otimes \sigma)$ (i.e. $\sigma$ is a morphism of algebras)

(2) $\mu_{E} \circ\left(\mu_{E} \otimes \mathrm{Id}\right)=\mu_{E} \circ\left(\mathrm{Id} \otimes \mu_{E}\right)$ (associativity)

Proof. The calculation

$$
\sigma \circ \mu_{E}=\sigma \circ \alpha \circ(\sigma \otimes \mathrm{Id})=\mu \circ(\operatorname{Id} \otimes \sigma) \circ(\sigma \otimes \mathrm{Id})=\mu \circ(\sigma \otimes \sigma)
$$

proves the first claim. The calculation

$$
\begin{aligned}
& \mu_{E} \circ\left(\operatorname{Id} \otimes \mu_{E}\right)= \\
& \quad \alpha \circ(\sigma \otimes \alpha \circ(\sigma \otimes \text { Id }))=\alpha \circ(\mu \circ(\sigma \otimes \sigma) \otimes \text { Id })= \\
& \alpha \circ\left(\left(\sigma \circ \mu_{E}\right) \otimes I d\right)=\mu_{E} \circ\left(\mu_{E} \otimes I d\right)
\end{aligned}
$$

proves the second claim.

It follows that $M$ is a two-sided ideal in $E$ (with respect to $\mu_{E}$ ).

Let $\left[, \uparrow:=\mu_{E}-\mu_{E} \circ \tau: E \otimes E \rightarrow E\right.$ be the corresponding commutator bracket. It follows from the associativity of $\mu_{E}$ that [, ${ }^{\curlyvee}$ satisfies the Jacobi identity, hence defines a structure of a Lie algebra on $E$.

It follow straight from the definition that the composition $M \otimes E \stackrel{\kappa \otimes I d}{\longrightarrow}$ $E \otimes E \stackrel{\mu_{E}}{\longrightarrow} E$ is equal to zero, hence

$$
\left[, \zeta \circ(\operatorname{Id} \otimes \kappa)=\mu_{E} \circ(\operatorname{Id} \otimes \kappa): E \otimes M \rightarrow M\right.
$$

We define $\mathrm{F}(E, \kappa, \sigma)=(\widehat{\mathfrak{g}}, \iota, \pi)$ as the pull-back of (4) by the canonical map $\mathfrak{g} \rightarrow U(\mathfrak{g})_{+}:$

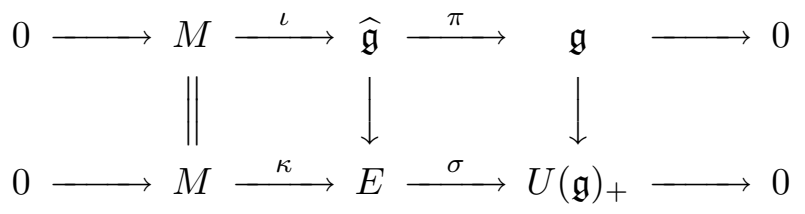

We will denote the induced Lie bracket on $\widehat{\mathfrak{g}}$ by $[, \uparrow$ as well.

It is clear that the assignment $(E, \kappa, \sigma) \mapsto \mathrm{F}(E, \kappa, \sigma)$ extends to a functor

$$
\mathrm{F}: \mathbb{E X T}_{U(\mathfrak{g})}^{1}\left(U(\mathfrak{g})_{+}, M\right) \rightarrow \operatorname{AbExt}(\mathfrak{g}, M)
$$

\subsection{Main theorem.}

Theorem 3.3. The functors (9) and (13)

$$
\mathrm{E}: \operatorname{AbExt}(\mathfrak{g}, M) \rightleftarrows \mathbb{E} \mathbb{X}_{U(\mathfrak{g})}^{1}\left(U(\mathfrak{g})_{+}, M\right): \mathrm{F}
$$

are mutually (quasi)inverse equivalences of categories. 
Proof. We fix $(E, \kappa, \sigma) \in \mathbb{E X}_{U(\mathfrak{g})}^{1}\left(U(\mathfrak{g})_{+}, M\right)$ for the time being and let $(\widehat{\mathfrak{g}}, \iota, \pi)=\mathrm{F}(E, \kappa, \sigma)$. Since the bracket $[, \widetilde{\jmath}$ is induced from the associative operation $\mu_{E}$, the map $\widehat{\mathfrak{g}} \rightarrow E$ extends canonically to a morphism of associative algebras

$$
\varphi: U(\widehat{\mathfrak{g}})_{+} \rightarrow E .
$$

The same applies to the morphism $\mathfrak{g} \rightarrow U(\mathfrak{g})_{+}$, giving rise to the identity map, and the naturality implies that the composition $U(\widehat{\mathfrak{g}})_{+} \rightarrow E \stackrel{\sigma}{\rightarrow} U(\mathfrak{g})$ is equal to the morphism (induced by) $\pi$.

We claim that the composition

$$
U(\widehat{\mathfrak{g}}) \otimes M \stackrel{\operatorname{Id} \otimes \iota}{\longrightarrow} U(\widehat{\mathfrak{g}}) \otimes U(\widehat{\mathfrak{g}})_{+} \stackrel{\widehat{\mu}}{\longrightarrow} U(\widehat{\mathfrak{g}})_{+} \stackrel{\varphi}{\longrightarrow} E
$$

is equal to the composition

$$
U(\widehat{\mathfrak{g}}) \otimes M \stackrel{\widehat{\mathrm{ad}}}{\longrightarrow} M \stackrel{\kappa}{\rightarrow} E .
$$

Indeed, since $\varphi$ is a morphism of algebras and $\kappa=\varphi \circ \iota$, the composition (14) is equal to the composition

$$
U(\widehat{\mathfrak{g}}) \otimes M \stackrel{\varphi \otimes \mathrm{Id}}{\longrightarrow} E \otimes M \stackrel{\operatorname{Id} \otimes \kappa}{\longrightarrow} E \otimes E \stackrel{\mu_{E}}{\longrightarrow} E
$$

Since $M$ is an ideal in $E$, the composition (15) factors through $\kappa$ :

$$
U(\widehat{\mathfrak{g}}) \otimes M \stackrel{\varphi \otimes \mathrm{Id}}{\longrightarrow} E \otimes M \stackrel{\widetilde{\mu_{E}}}{\longrightarrow} M \stackrel{\kappa}{\rightarrow} E
$$

The morphism $\widetilde{\mu_{E}} \circ(\varphi \otimes$ Id $)$ defines a structure of a $U(\widehat{\mathfrak{g}})$-module on $M$ which is determined by its restriction to $\widehat{\mathfrak{g}}$.

By (11), the composition

$$
\widehat{\mathfrak{g}} \otimes M \stackrel{\varphi \otimes \mathrm{Id}}{\longrightarrow} E \otimes M \stackrel{\widetilde{\mu_{E}}}{\longrightarrow} M
$$

is equal to the composition

$$
\widehat{\mathfrak{g}} \otimes M \stackrel{\varphi \otimes \mathrm{Id}}{\longrightarrow} E \otimes M \stackrel{\left[\widetilde{[,]^{\mu}}\right.}{\longrightarrow} M
$$

which is precisely the restriction of the adjoint representation to $M$.

It follows that the morphism $\varphi$ gives rise to an isomorphism

$$
\mathrm{E}(\mathrm{F}(E, \kappa, \sigma)) \rightarrow(E, \kappa, \sigma) .
$$

The naturality of the construction shows that, in fact, it gives rise to an isomorphism $\mathrm{E} \circ \mathrm{F} \rightarrow \mathrm{Id}$.

Now, we fix $(\widehat{\mathfrak{g}}, \iota, \pi) \in \operatorname{AbExt}(\mathfrak{g}, M)$ and let $(E, \kappa, \sigma)=\mathrm{F}(\widehat{\mathfrak{g}}, \iota, \pi)$. By the definition of $E$ we have a morphism $\varphi: U(\widehat{\mathfrak{g}})_{+} \rightarrow E$ such that the composition

$$
\widehat{\mathfrak{g}} \rightarrow U(\widehat{\mathfrak{g}})_{+} \stackrel{\varphi}{\rightarrow} E \stackrel{\sigma}{\rightarrow} U(\mathfrak{g})_{+}
$$

São Paulo J.Math.Sci. 6, 1 (2012), 71-80 
is equal to the composition

$$
\widehat{\mathfrak{g}} \rightarrow U(\widehat{\mathfrak{g}})_{+} \stackrel{\pi}{\rightarrow} U(\mathfrak{g})_{+}
$$

and, therefore, we have the canonical morphism

$$
\widehat{\mathfrak{g}} \rightarrow E \times \times_{U(\mathfrak{g})_{+}} \mathfrak{g}
$$

which is easily seen to be an isomorphism. It remains to show that the morphism (16) is a morphism of Lie algebras. For this it suffices to show that the morphism $\varphi$ is a morphism of algebras with the respect to the product $\mu_{E}$ on $E$ defined by (10) and the product on $U(\widehat{\mathfrak{g}})_{+}$which we denote by $\widehat{\mu}$.

Since $\varphi$ is a morphism of $U(\widehat{\mathfrak{g}})$-modules we have $\varphi \circ \widehat{\mu}=\alpha \circ(\pi \otimes \varphi)$. Hence,

$$
\mu_{E} \circ(\varphi \otimes \varphi)=\alpha \circ((\sigma \circ \varphi) \otimes \varphi)=\alpha \circ(\pi \otimes \varphi)=\varphi \circ \widehat{\mu} .
$$

It follows that the morphism (16) gives rise to an isomorphism

$$
(\widehat{\mathfrak{g}}, \iota, \pi) \rightarrow \mathrm{F}(\mathrm{E}(\widehat{\mathfrak{g}}, \iota, \pi))
$$

The naturality of the construction shows that we have an isomorphism of functors $\mathrm{Id} \rightarrow \mathrm{F} \circ \mathrm{E}$.

\section{Linear algebra}

In this section we sketch the relationship of the constructions of Section 3 to the respective monoidal structures on $\operatorname{AbExt}(\mathfrak{g}, M)$ and $\mathbb{E} \mathbb{X T}_{U(\mathfrak{g})}^{1}\left(U(\mathfrak{g})_{+}, M\right)$

The monoidal structure structure on both $\operatorname{AbExt}(\mathfrak{g}, M)$ and $\mathbb{E X T}_{U(\mathfrak{g})}^{1}\left(U(\mathfrak{g})_{+}, M\right)$ is induces by the Baer sum of extensions and will be denoted + in both cases.

Suppose given $\left(\widehat{\mathfrak{g}}_{i}, \pi_{i}, \iota_{i}\right) \in \operatorname{AbExt}(\mathfrak{g}, M), i=1,2$. Let $\left(E_{i}, \kappa_{i}, \sigma_{i}\right)=$ $\mathrm{E}\left(\widehat{\mathfrak{g}}_{i}, \pi_{i}, \iota_{i}\right)$.

Let $\mathfrak{G}_{i}$ denote the cone of $\iota_{i}$. Note that $\operatorname{Cone}\left(M \times M \stackrel{\iota_{1} \times \iota_{2}}{\longrightarrow} \widehat{\mathfrak{g}}_{1} \times \mathfrak{g}\right.$ $\left.\widehat{\mathfrak{g}}_{2}\right)=\mathfrak{G}_{1} \times_{\mathfrak{g}} \mathfrak{G}_{2}$. The projection $\mathrm{pr}_{i}: \mathfrak{G}_{1} \times_{\mathfrak{g}} \mathfrak{G}_{2} \rightarrow \mathfrak{G}_{i}$ give rise to the map $\operatorname{pr}_{i}: U\left(\mathfrak{G}_{1} \times_{\mathfrak{g}} \mathfrak{G}_{2}\right) \rightarrow U\left(\mathfrak{G}_{i}\right)$. The composition $\mathfrak{G}_{1} \times_{\mathfrak{g}} \mathfrak{G}_{2} \rightarrow \mathfrak{G}_{i} \rightarrow \mathfrak{g}$ is independent of $i$, therefore, so is the composition $U\left(\mathfrak{G}_{1} \times \mathfrak{g} \mathfrak{G}_{2}\right) \rightarrow U\left(\mathfrak{G}_{i}\right) \rightarrow$ $U(\mathfrak{g})$. Hence, the maps $\operatorname{pr}_{i}$ give rise to the map

$$
U\left(\mathfrak{G}_{1} \times_{\mathfrak{g}} \mathfrak{G}_{2}\right) \rightarrow U\left(\mathfrak{G}_{1}\right) \times_{U(\mathfrak{g})} U\left(\mathfrak{G}_{2}\right)
$$


of DGA resulting in the commutative diagram

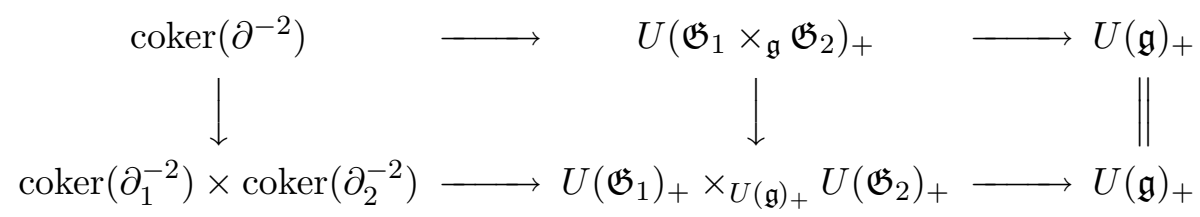

Commutativity of the diagram

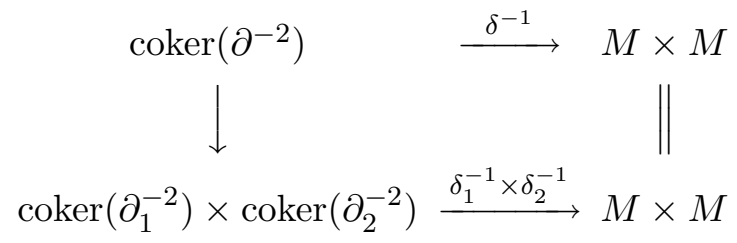

shows that the map (17) gives rise to the morphism of extensions

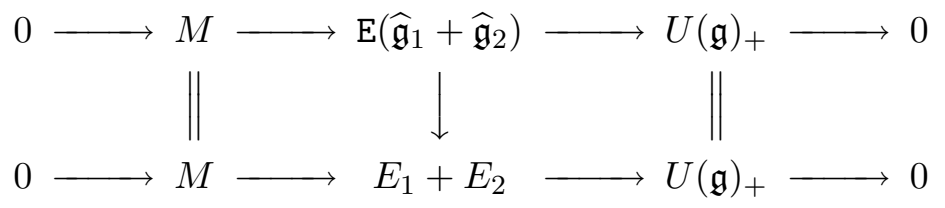

The evident naturality of the above construction mean that is extends to an isomorphism of bi-functors $\mathrm{E} \circ(\cdot+\cdot) \rightarrow(\cdot+\cdot) \circ(\mathrm{E} \times \mathrm{E})$. It is easy (albeit tedious) to show that the latter endows $\mathrm{E}$ with a structure of a monoidal functor. We leave the details to the reader.

The functor $\mathrm{F}$ is defined by pull-back functor at the level of the underlying extensions. The latter is equipped with the canonical structure of a monoidal functor. It is easy to see that this structure is compatible with Lie brackets. It is easy to check that the natural transformations $\mathrm{E} \circ \mathrm{F} \rightarrow \mathrm{Id}$ and $\mathrm{Id} \rightarrow \mathrm{F} \circ \mathrm{E}$ constructed in the course of the proof of Theorem 3.3 are morphisms of monoidal functors.

\section{References}

[CE] H. Cartan, S. Eilenberg, Homological Algebra, Princeton University Press, Princeton NJ, 1999

[M] S. MacLane, Categories for the working mathematician, Graduate Texts in Mathematics, vol. 5, 2nd edition, Springer-Verlag, New York, 1998, 